\title{
Optimal and near-optimal exponent-pairs for the Bertalanffy- Pütter growth model
}

Katharina Renner-Martin ${ }^{\text {Corresp., }}{ }^{1}$, Norbert Brunner ${ }^{1}$, Manfred Kühleitner ${ }^{1}$ ， Werner-Georg Nowak ${ }^{1}$, Klaus Scheicher ${ }^{1}$

${ }^{1}$ Department of Integrative Biology and Biodiversity, Institute of Mathematics, Universität für Bodenkultur Wien, Vienna, Austria

Corresponding Author: Katharina Renner-Martin

Email address: kathi.renner-martin@gmx.de

The Bertalanffy-Pütter growth model describes mass $m$ at age $t$ by means of the differential equation $d m / d t=p^{*} m^{\wedge} a-q^{*} m^{\wedge} b$. The special case using the von Bertalanffy exponent-pair $a=2 / 3$ and $b=1$ is most common (it corresponds to the von Bertalanffy growth function VBGF for length in fishery literature). Fitting VBGF to size-at-age data requires the optimization of three model parameters (the constants $p, q$, and an initial value for the differential equation). For the general Bertalanffy-Pütter model, two more model parameters are optimized (the pair $a<b$ of non-negative exponents). While this reduces bias in growth estimates, it increases model complexity and more advanced optimization methods are needed, such as the Nelder-Mead amoeba method, interior point methods, or simulated annealing. Is the improved performance worth these efforts? For the case, where the exponent $b=1$ remains fixed, it is known that for most fish data any exponent $\mathrm{a}<1$ could be used to model growth without affecting the fit to the data significantly (when the other parameters were optimized). We hypothesized that the optimization of both exponents would result in a significantly better fit of the optimal growth function to the data and we tested this conjecture for a data set $(20,166$ fish) about the mass-growth of Walleye (Sander vitreus), a fish from Lake Erie, USA. To this end, we assessed the fit on a grid of 14,281 exponent-pairs $(a, b)$ and identified the best fitting model curve on the boundary $a=b$ of the grid $(a=b=0.686)$; it corresponds to the generalized Gompertz equation $d m / d t=p^{*} m^{\wedge} a-q^{*} \ln (m)^{*} m^{\wedge} a$. Using the Akaike information criterion for model selection, the answer to the conjecture was no: The von Bertalanffy exponent-pair model (but not the logistic model) remained parsimonious. However, the bias reduction attained by the optimal exponent-pair may be worth the tradeoff with complexity in some situations where predictive power is solely preferred. Therefore we recommend the use of the Bertalanffy-Pütter model (and of its limit case, the generalized Gompertz model) in natural resources management (such as in fishery stock assessments), as it relies on careful quantitative assessments to recommend policies for 
sustainable resource usage. 


\section{Optimal and near-optimal exponent-pairs for the Bertalanffy-Pütter growth model}

2 Katharina Renner-Martin, Norbert Brunner, Manfred Kühleitner, Werner-Georg Nowak, and Klaus

3 Scheicher

4

5 Institute of Mathematics, Department of Integrative Biology and Biodiversity Research, University of 6 Natural Resources and Life Sciences, Gregor Mendel Strasse 33, A-1180 Vienna, Austria

7

8 Corresponding author:

9 Katharina Renner-Martin

10 E-mail: kathi.renner-martin@gmx.de

\section{Statements}

13 The authors declare no conflict of interests.

14 The first author was supported by a grant from the University of Natural Resources and Life Sciences, 15 Vienna. 
17 Optimal and near-optimal exponent-pairs for the Bertalanffy-Pütter growth model

18 Abstract. The Bertalanffy-Pütter growth model describes mass $m$ at age $t$ by means of the differential equation $d m / d t=p \cdot m^{a}-q \cdot m^{b}$. The special case using the von Bertalanffy exponent-pair $a=2 / 3$ and $b=1$ is most common (it corresponds to the von Bertalanffy growth function VBGF for length in fishery literature). Fitting VBGF to size-at-age data requires the optimization of three model parameters (the constants $p, q$, and an initial value for the differential equation). For the general Bertalanffy-Pütter model, two more model parameters are optimized (the pair $a<b$ of non-negative exponents). While this reduces bias in growth estimates, it increases model complexity and more advanced optimization methods are needed, such as the Nelder-Mead amoeba method, interior point methods, or simulated annealing. Is the improved performance worth these efforts? For the case, where the exponent $b=1$ remains fixed, it is known that for most fish data any exponent $a<1$ could be used to model growth without affecting the fit to the data significantly (when the other parameters were optimized). We hypothesized that the optimization of both exponents would result in a significantly better fit of the optimal growth function to the data and we tested this conjecture for a data set (20,166 fish) about the mass-growth of Walleye (Sander vitreus), a fish from Lake Erie, USA. To this end, we assessed the fit on a grid of 14,281 exponent-pairs $(a, b)$ and identified the best fitting model curve on the boundary $a=b$ of the grid ( $a=b=$ 0.686); it corresponds to the generalized Gompertz equation $d m / d t=p \cdot m^{a}-q \cdot \ln (m) \cdot m^{a}$. Using the Akaike information criterion for model selection, the answer to the conjecture was no: The von Bertalanffy exponent-pair model (but not the logistic model) remained parsimonious. However, the bias reduction attained by the optimal exponent-pair may be worth the tradeoff with complexity in some situations where predictive power is solely preferred. Therefore, we recommend the use of the Bertalanffy-Pütter model (and of its limit case, the generalized Gompertz model) in natural resources management (such as in fishery stock assessments), as it relies on careful quantitative assessments to recommend policies for sustainable resource usage.

Subjects Computational Biology, Aquaculture (Fisheries and Fish Science), Mathematical Biology,

42 Computational Science

43

Keywords Bertalanffy-Pütter differential equation, Akaike information criterion $(A I C)$, Region of nearoptimality

\section{INTRODUCTION}

Size-at-age (length or mass) is an important metric about animals (Google search: ca. 286,000 results), in particular for fisheries management (Ogle \& Iserman, 2017). Consequently, various models for size-at-age have been proposed, whereby models aiming at biological explanations for growth focus on mass-at-age (c.f. Ursin, 1979; Enberg et al., 2008). Here, we investigate a general class of growth models in terms of the von Bertalanffy (1957) and Pütter (1920) differential equation (1):
(1) $\frac{d m(t)}{d t}=p \cdot m(t)^{a}-q \cdot m(t)^{b}$. 
53 Equation (1) describes body mass (weight) $m(t)>0$ as a function of age $t$, using five model

54 parameters: $a, b, p, q, m_{0}$. Thereby, $m_{0}>0$ is an initial value, i.e. $m(0)=m_{0}$. The exponent-pair $a$

$55<b$ ('metabolic scaling exponents') is non-negative and also the constants $p$ and $q$ are non-

56 negative. Several 'named models' are special instances of (1): To describe mass-at-age,

57 Bertalanffy (1957) suggested the exponent-pair $a=2 / 3$ and $b=1$, West, Brown \& Enquist

58 (2001) proposed $a=3 / 4, b=1$, other authors considered $a=1, b=2$ (logistic growth of

59 Verhulst, 1838), Richards (1959) recommended $a=1$ while retaining $b>1$ as a free parameter,

60 and the generalized Bertalanffy growth model assumes $b=1$, using $a<1$ as parameter

61 (recommended e.g. by Pauly, 1981). There are also models of type (1) for length-at-age, notably

62 VBGF, the von Bertalanffy growth function with exponent-pair $a=0, b=1$ (bounded

63 exponential growth) which is widely used in fishery literature (Google search for 'VBGF, fish':

64 ca. 15,000 results). VBGF is equivalent to the model with the von Bertalanffy exponent-pair ( $a=$

$652 / 3, b=1$ ) for mass-growth (Bertalanffy, 1957).

66 In the case of equal exponents, the generalized Gompertz differential equation (2) replaces

67 equation (1). Its right hand side is the limit of the right hand side of (1), assuming $b$ approaches

68 a. Its special case $a=1$ defines the Gompertz (1832) model;

69

(2) $\frac{d m(t)}{d t}=p \cdot m(t)^{a}-q \cdot \ln (m(t)) \cdot m(t)^{a}$.

70 In general, the solutions of (1) and (2) involve non-elementary functions, namely hypergeometric

71 functions and exponential integrals, respectively (Ohnishi, Yamakawa \& Akamine, 2014;

72 Marusic \& Bajzer, 1993; further explanations: Seaborn, 2013). The solutions of the more special

73 'named models' are elementary. 
74 Concrete values for the parameters of equations (1), (2) are obtained by identifying a growth 75 function (i.e. a concrete solution of the differential equations) with the best fit to the data. 76 Experience has shown that no single of the above-mentioned 'named models' was exactly 77 correct for all species (c.f. Killen, Atkinson \& Glazier, 2010 for fish; White, 2010 for mammals). Renner-Martin et al. (2018) explored the situation for the generalized von Bertalanffy model (the exponent $b=1$ is held fixed) and found that for most species of fish there was a high variability, meaning that any exponent (i.e. $0 \leq a<1$ ) could be used to model growth without affecting the fit to the data significantly (when the other parameters $p, q, m_{0}$ were optimized). They explained this by data quality, as for wild-caught fish and also for wildlife data there is always the problem of 'haphazard' sampling, which may result in unreliable growth parameter estimates (Wilson et al., 2015).

Is this high variability for fish data still observed, if both exponents $(a, b)$ of the Bertalanffy-

86 Pütter model are optimized? We explore the region of near-optimal exponent-pairs and

87 hypothesize that the additional degree of freedom for the optimization of the exponent-pair 88 (instead of the optimization of one exponent, only) would result in a significantly better fit of the optimal growth function and thus in a small region of near-optimality. Thereby, the term 'nearoptimal' (see the Discussion) may be defined by different measures of the goodness of fit; here we consider two such measures: One is the sum of squared errors ( $S S E)$, which comes from the

92 most common approach to data fitting, the method of least squares. The other is the Akaike 93 weight, which comes from the theory of model selection by means of the Akaike' (1974) 94 information criterion $A I C$; for a discussion of alternative information measures c.f. Dziak et al. 95 (2017). 
96

97

98

99

100

101

102

103

104

105

106

107

108

109

110

111

112

113

114

115

116

117

118

\section{MATERIALS AND METHODS}

\section{Study overview}

We started with a literature search for mass-at-age data of fish. These data are exceptional, as most growth data for fish are length-at-age. In view of the computational complexity of optimizing the Bertalanffy-Pütter-model, we focus on one case study and identify optimal exponents for one fish data-set only.

Technically, given the data, we studied the function $\operatorname{SSE}_{\text {opt }}(a, b)$, which for each exponent-pair $(a, b)$ identifies the minimal $S S E$ that can be obtained optimizing the parameters $p, q, m_{0}$. As we aimed at evaluating and minimizing this target function $S S E_{\text {opt }}$ on a large grid of exponent-pairs, a fast and reliable optimization method was needed. We therefore started with several advanced general-purpose methods and a coarse grid in order to obtain a rough idea about the shape of $S S E_{\text {opt }}$ and the performance of the different methods. We then selected a method (interior point optimization) and applied it to a refined grid. Finally, we developed a custom-made method (based on simulated annealing) to identify the globally optimal exponent-pair (which no longer needed to be a grid point). As a complication, for the chosen data also the boundary diagonal $a=$ $b$ of the parameter region (i.e. $0 \leq a<b$ ) needed to be considered, whence the same computations were repeated for this diagonal. This search of optimal parameters for equations (1) and (2) used Mathematica 11.3.

For a given exponent-pair $(a, b)$, we then assessed the goodness of the fit of its optimal model curve to the data in relation to the globally optimal exponent pair (and its best-fitting model curve). We plotted the respective model curves, compared $\operatorname{SSE}_{\text {opt }}(a, b)$ with the minimal value of $S S E_{\text {opt }}$, and used this information to compute also the respective values of $A I C$ and the Akaike weights. We outline the details of our methodological approach below. There we mention also 
119 alternative approaches (i.e. different definitions of the target function). With respect to the results

120 of this paper, we expected that different approaches might result in different optimal exponent-

121 pairs (because then a different function is optimized). However, we expected that the general

122 feature of the present optimization problem, such as the flatness of the target function in a large

123 neighborhood of the optimal exponent-pair (whence e.g. elementary methods of optimization

124 may fail) will persist independently of the other methodological details. We therefore selected a 125 relatively elementary approach that nevertheless could be adapted to any of the other mentioned 126 methodological variants.

\section{Data}

128 We used 'FSAdata WalleyeErie2'from Ogle (2018) about Walleye (Sander vitreus) from Lake 129 Erie, USA, and retrieved a sub-sample (20,166 data-points) about male fish. The data informed about mass (in gram) and age (in years from otholits) of wild-caught fish. We applied MS Excel

131 to retrieve the data and to pivote them (i.e. to identify average weights for the age classes).

132 Figure 1 plots the data and the average weights.

133 There were few data about young fish (14 of age 0$)$ and likewise few about older fish (22 with 134 age 16-20 years), and none about fish with age 21-29 (maximal observed age reported in 135 FishBase: Froese \& Pauly, 2018). This may indicate gear bias (where small or large fish were not 136 adequately sampled). In order to obtain more balanced class-sizes, smaller classes were merged; 137 the outcome is Table 1 , reporting of each class the average mass at the average age. Thus, 13 138 classes representing larger samples were evaluated instead of originally 20 age classes.

139 At first it may appear troubling to take more than 20,000 data points and then aggregate them to merely 13 mass-at-age classes. However, for data fitting it was the distance between the model

141 curve and the average of each class that mattered. The distances between the average and the 
142 other class data could not be improved by a growth model. Further, in view of the large sample

143 size it was reasonable to assume that average mass might be normally distributed (even if mass

144 itself might follow another distribution), as in general average values are asymptotically

145 normally distributed. (However, we do not use this assumption.)

\section{General approach to data fitting}

147 Numerical instability tends to impede fitting the generalized Bertalanffy model (i.e. optimization

148 of $a, p, q, m_{0}$, when $b=1$ ) to noisy data (Shi et al. 2014). Even for simple models (meaning:

149 certain values for the exponents are assumed and the other three parameters are optimized)

150 literature reported that optimization failed to converge for certain data sets (Apostolidis \&

151 Stergiou, 2013). One of the reasons was the use of parametrizations that require bounded growth

152 functions (e.g. Cailliet et al., 2006), whereas not all data may support bounded growth. Another

153 reason was the observation that even for simple models the problem of data fitting may overtask

154 straightforward optimization routines. Clearly, with more parameters to optimize the problem of

155 convergence becomes more demanding and also powerful methods slow down. In order to avoid

156 running into numerical instability by the use of too many parameters, we used a grid search,

157 where for each grid-point (exponent-pair $a, b)$ we identified model parameters $\left(p, q, m_{0}\right)$ that

158 minimized the following function:

159

(3) $S S E_{\text {opt }}(a, b)=\min _{m_{0}, p, q}(S S E)$ for growth functions with exponents $a, b$.

160 There are various improvements of regression models, such as mixed-effect models to identify

161 explanatory factors for growth (Strathe et al., 2010). However, such models require highly

162 controlled experiments, whereas the present data are about wild-caught fish with unknown life

163 history. In view of the difficulties with the convergence of optimization we did not consider 
164 more complex model assumptions, such as heteroscedastic growth that assumes a larger variance 165 for a higher mass, or models that need additional parameters to distinguish different growth 166 phases (Manabe et al., 2018). Further, in order to allow a meaningful visual comparison of the 167 goodness fit of different model by an inspection of the plotted model curves, we did not use 168 weighted sums of squared errors. (Examples for weights used in literature are the counts of fish 169 at age and the reciprocals of the standard deviations of their masses.) For otherwise, a curve 170 whose plot appears to fit well may actually fit poorly, if it misses a heavily weighed data point. 171 Thus, the purpose of optimization was the identification of a suitable growth curve for the 172 considered species and not the identification of a growth curve that would minimize errors in relation to a given population. We did not simplify optimization by adding assumptions about

174 parameter values, e.g. eliminating two parameters from optimization by using a literature value 175 for the initial condition $m_{0}$ (rather than optimizing it) and using a literature value for the 176 asymptotic mass (defined below). In this case $\operatorname{SSE}_{o p t}(a, b)$ could have been computed very fast 177 from the optimization of one parameter, only, but at the cost of weakening the link to the data.

178 The use of grid-points helped to identify failures of optimization by a visual inspection (e.g. a 179 grid-point with exceptionally high $S S E_{\text {opt }}$, when compared to neighboring grid-points). In order 180 to do not miss the optimum, we used different approaches to data-fitting to identify and correct miscalculations. Thereby, computation time was an issue. For instance, commercially available software packages for fisheries management use powerful numerical methods to determine the model parameters even for the simple models (Mildenberger et al., 2017). These methods aim at optimizing one given model, where computing time is not an issue. Instead, here we aimed at optimizing a large number of models simultaneously in order to explore the function $S S E_{\text {opt }}$; i.e. each grid point defined a model (defined from the exponent pair $a, b$ ) for which optimal 
187 parameters were identified. While for each grid-point $S S E_{\text {opt }}$ could be obtained fast, optimizing

188 over the whole grid was time consuming. For example, covering the region $0 \leq a \leq 1, a<b \leq 3$

189 by a grid with neighboring points at distance 0.01 would define 25,250 grid points. For this grid,

190 assuming six optimizations per minute would require 70 hours of computing (CPU) time.

191 Optimization proceeded in three stages. First, $S S E_{\text {opt }}$ was computed on a coarse grid (step-size

192 0.1) to sketch the shape of $S S E_{\text {opt }}$ and locate a region of near-optimal exponents. This used

193 methods of optimization that were fast, but not necessarily accurate. In the second stage, the

194 computations were repeated with a finer grid (step-size 0.01) and using more accurate methods

195 of optimization. These computations allowed to identify candidates for the optimum. In the final

196 stage a search for the global optimum was performed, starting with these candidate points. The

197 specific methods of optimization used in each step are explained below (c.f. the survey of

198 Cedersund et al., 2015).

199 In order to speed up computations all approaches solved the differential equations (1) and (2) 200 numerically (Leader, 2004). Using the analytic solutions of the differential equations (these are 201 available in Mathematica) would make data fitting time consuming even for a given exponent 202 pair. As the numerical methods used by Mathematica 11.3 work with high precision, this did not 203 compromise the accuracy of optimization.

\section{Starting values for data fitting}

205 For most iterative methods of optimization, reasonable starting values for the parameters are 206 needed to ensure convergence of optimization. For instance, the starting value for the initial 207 value $m_{0}$ was the first data point of Table 1. 
208 For the other parameters, practitioners use various rules of thumb (Carvalho \& Santoro, 2007),

209 which utilize general considerations about the possible shape of the growth functions. For, the 210 typical solutions of (1) and (2) are increasing, bounded and sigmoidal. (However, there are also 211 non-sigmoidal solutions, e.g. $a=0$, and unbounded solutions, e.g. $q=0$ and $p>0$.) Initially the 212 rate of growth increases, until the inception point is reached. Subsequently it decreases to zero in 213 the limit, when the asymptotic mass $m_{\max }$ is reached; there the right-hand side of (1) and (2), 214 respectively, vanishes. For equation (1) with $a<b$ this results in the following equation: (4) $m_{\max }=\left(\frac{p}{q}\right)^{\frac{1}{b-a}}$. To obtain a starting value $q_{0}$ for the parameter $q$, we assumed for the moment that the asymptotic mass would exceed the maximal observed mass by $20 \%$, i.e. we solved the equation $m_{\max }=$ $1.2 \cdot \max (m)$ for $q$, referring to equation (4). This resulted in $q_{0}=p_{0} /(1.2 \cdot \max (m))^{b-a}$, where $p_{0}$ was the starting value for $p$.

In order to obtain a starting value for $p$, we evaluated equation (1) approximately at $t=0$, using for the right-hand side the above-mentioned starting value $m_{0}$ for $m$ and $q_{0}$ for $q$. As approximate value for the derivative, $m^{\prime}(0)$, we used the derivative at $t=0$ of the quadratic interpolation polynomial (Burden \& Faires, 1993) through the first three points listed in Table 1. This polynomial was an approximation for the growth function in the neighborhood of $t=0$. Solving

$$
\text { (5) } p_{0}=\frac{m^{\prime}(0) \cdot 1.2^{b} \cdot \max (m)^{b}}{1.2^{b} \cdot \max (m)^{b} \cdot m_{0}^{a}-1.2^{a} \cdot \max (m)^{a} \cdot m_{0}^{b}} \text {. }
$$

227 These formulas defined starting values for $m_{0}, p$ and $q$. The formulas were problematic for exponents close to the diagonal, as the function $p_{0}$ tends to infinity in the limit $a \rightarrow b$. Therefore, 
229 for exponents $b=a+0.01$ we used simulated annealing (see below) in case that optimization

230 using these starting values did not converge.

231 Preparatory screening

$232 S S E_{\text {opt }}$ was computed for a coarse grid (distance 0.1 between adjacent points), using two general 233 purpose methods for global optimization in parallel, simulated annealing and the Nelder-Mead 234 amoeba method. Both methods are available for the Mathematica function NMinimize.

235 We used simulated annealing, as we expected it to produce reasonable results. It used random 236 numbers as starting values (using multiple starting values) and then altered them by random 237 fluctuations, accepting parameters with lower values of $S S E$, but also accepting with a certain 238 probability (that became lower in subsequent iteration steps) parameters with a higher SSE to escape from suboptimal local extrema (Vidal, 1993). In order to ensure replicability, the default random seed 0 was used. Therefore, if $S S E$ was optimized repeatedly for the same grid-point, the outcome remained the same.

242 We used the amoeba method, because it is fast. Given the exponent-pair $a, b$, the method first evaluates four corners of a tetrahedron (simplex) in parameter space (dimensions $m_{0}, p, q$ ) and successively applies reflections (moving the point with highest SSE through the opposite side of the tetrahedron to a point with perhaps lower SSE) and shrinking (zooming in to a local 246 minimum point).

247 In order to avoid obviously meaningless parameter values, we added constraints to ensure an in 248 relation to the data biologically reasonable initial value $m_{0}>10$ and positive parameters $p>q$. 


\section{Semi-automated optimization}

250 In order to employ also methods developed specifically for the least squares method, we used an

251 alternative approach using the Mathematica function NonlinearModelFit. It implements the most

252 common methods for nonlinear regression.

253 The optimization loop assumed a fixed value for $a$, whereas $b$ proceeded from $b=a+0.01$ to $b=$

2542 with step size 0.01 . Further, for each exponent $a=n \cdot 0.01$ we plotted the hitherto obtained

255 values of $\operatorname{SSE}_{\text {opt }}(a, b)$. If the plot showed a U-shape, then we could identify a minimum of SSE

256 on the line $a=n \cdot 0.01, b>a$; otherwise (human intervention) we added more values of $b$ to the

257 loop until we could discern the U-shape. (We thereby assumed that for still larger exponents $b$

258 the fit could only become worse. This assumption was corroborated by the initial screening.)

259 The optimization started at $a=0, b=0.01$ with initial values for $m_{0}, p$ and $q$ explained above.

260 For the subsequent computations, where $a$ was kept fixed and $b$ moved, the iterative optimization

261 at the next $b$, namely at $b+0.01$, started with the optimal parameters from the previous

262 optimization (for $b$ ).

263 However, in order to ensure convergence (and an empirically meaningful outcome), we

264 minimized SSE subject to certain constraints $\left(m_{0}>10\right.$ and $\left.q>0\right)$, whence many common

265 methods from regression analysis (e.g. Levenberg-Marquardt algorithm) were not applicable.

266 Instead, we used an interior point method. These methods (e.g. barrier methods initially

267 developed in the 1960s) became popular in 1984, when an interior point method (Karmakar,

268 1984) solved linear optimization problems in polynomial time; Forsgen, Gill \& Wright (2002)

269 refer to the 'interior point revolution'. This setting was also advantageous for the present 270 problem. 


\section{Custom-made simulated annealing}

272 Based on this preparatory work, we could evaluate $\operatorname{SSE}_{\text {opt }}(a, b)$ for almost all grid points. In order

273 to improve the estimates of $S S E$ at the best fitting grid points and to move from there to the

274 optimal exponent-pair (no longer a grid-point), we developed a custom-made approach of

275 simulated annealing. (We used the general purpose method of Mathematica in the preparatory

276 screening, but its performance was suboptimal, whence modifications were needed to ensure

277 convergence in reasonable time.) The main difference to general purpose simulated annealing

278 was the use of a (sort of) geometric Brownian motion. (For each step, rather than adding a small

279 random number to the parameters, they were multiplied by a random number, whence positive

280 values were retained.) The optimization used a loop with 500,000 steps: It started with the

281 parameter values obtained from the preparatory optimization steps.

282

283

284

285

286

287

\section{RESUltS}

Table 2 lists the optimal parameters for three exponent-pairs, Bertalanffy, logistic, and the optimal pair (of Gompertz-type), and Figure 2 visualizes the model curves defined from these optimal parameters; all model curves remained reasonably close to the data. (Failing optimizations often converged to a curve close to the mean value of the average masses.) The following optimization aimed at finding improvements of $S S E_{\text {opt }}=23,709$ for the Bertalanffypair, which was obtained in the initial round of optimizations.

First round of optimization: We evaluated $S S E_{\text {opt }}$ at grid-points $0 \leq a \leq 1$ and $a<b \leq 1.5$, for growth functions (1) and at $0 \leq a=b \leq 1$ for (2). These grid-points were exponent-pairs at distance 0.1 between successive grid-points. For each grid-point the better of the outcomes from (general purpose) simulated annealing and from the amoeba method was used; $\operatorname{SSE} E_{\text {opt }}(0.7,0.7)=$ 21,310 was optimal. However, the initial optimization became problematic for $b>1.2$ and it did 
294 not allow to decide, if optimization would require a search in this problematic region. Further, it

295 could not be decided, if the optimum would be located on or above the diagonal.

296 Second round of optimization: We conducted a systematic search (semi-automated data fitting)

297 confined to equation (1). It used a fine grid (distance 0.01 between successive exponent-pairs),

298 aiming at identifying for each exponent $a$ with $0 \leq a \leq 1$ an exponent $b>a$ with minimal $S S E$. (It

299 was sufficient to screen exponents $b \leq 2$.) The improved accuracy of this search was

300 demonstrated for the Bertalanffy exponent-pair with a lower $\operatorname{SS} E_{\text {opt }}(0.67,1)=23,534.6$. Figure 3

301 plots the outcome from the optimization at 14,282 grid points (exponent-pairs). The black dots

302 indicate, for each exponent $a$, for which exponent $b$ the value of SSE was minimal. Thereby,

$303 S S E_{\text {opt }}(0.67,0.7)=21,287.1$ was the least observed optimized $S S E$ for equation (1). This

304 demonstrates that optimization showed the following pattern: For $a=0$ the minimum $S S E$ was

305 reached close to $b=2$. For the following values there was a distinct U-shape to be observed till $a$

$306=0.67$. Finally, the optimum was attained close to the diagonal $a=b$ (dots moving upwards), but

307 the optimum value was increasing compared to the previous ones. This pattern supported the

308 hypothesis that the optimal $S S E$ would be attained within the (topological closure of the) search

309 region. However, the computations did not allow to decide, whether the global minimum of SSE

310 was attained for $b>a$, i.e. for equation (1), or for $b=a$, i.e. equation (2). Further, optimization

311 proceeded smoothly till $a=0.7$, but for larger exponents optimization became increasingly more

312 difficult and fewer results could be accepted. In particular, grid points near the diagonal were

313 problematic.

314 Third round of optimization: We tackled these issues using a global optimization. It started with

315 the near-optimal parameters found previously. For equation (1), starting from $a=0.68$ and $b=$

3160.69 , the least $S S E_{\text {opt }}(0.666703,0.705181)=21,287.5$ was achieved. However, for equation (2), 
317 i.e. on the diagonal $a=b$, a slightly better outcome $S S E_{\text {opt }}(0.686028,0.686028)=21,286.4$ was

318 obtained (parameters in Table 2). The custom-made method of simulated annealing improved

319 insofar upon the same method as implemented by Mathematica (which was used in the initial

320 step), as it was more accurate. Further, despite the high number of computing steps its

321 performance was more reliable (no unexpected computer crashes).

322 Summarizing, during the three rounds of optimization the fit achieved by the Bertalanffy 323 exponent-pair $\left(a=2 / 3, b=1\right.$ with $\left.S S E_{\text {opt }}=23,709\right)$ could be substantially improved. The first

324 round identified a better exponent-pair $\left(a=b=0.7\right.$ with $\left.S S E_{o p t}=21,310\right)$. The second round, 325 using more accurate computations, found a still better exponent pair $(a=0.67, b=0.7$ with $\left.326 S S E_{\text {opt }}=21,287.1\right)$. The final round converged to the minimal $S S E_{\text {opt }}=21,286.4$ at $a=b=$ 3270.686028 . Thus, by using different exponent-pairs and also by using more accurate optimization 328 methods, $S S E_{\text {opt }}$ could be reduced by $10 \%$ from the initial estimate using the von Bertalanffy 329 pair.

\section{DISCUSSION}

331 Optimization identified an exponent-pair that achieved a $10 \%$ reduction of $S S E_{\text {opt }}$, when compared to the von Bertalanffy-pair. Was this reduction worth the efforts? The answer to this question depends on what notion of 'near-optimality' is used (considered in this section) and for what purpose the model is needed (considered in the Conclusion).

An obvious definition of near-optimality would set a maximal percentage by which the optimal $S S E$ may be exceeded. However, the appropriate percentage may vary with the data. Here we explain a definition of near-optimality that refers to the Akaike weight; it therefore has the same meaning for all data. Specifically, we used an index $A I C_{c}$ for small sample sizes (Burnham \& Anderson, 2002; Motulsky \& Christopoulos, 2003). $A I C_{c}$ was defined from the least sum of 
340 squared errors for the model with exponent-pair $(a, b)$, i.e. $S S E$ (model) $=S S E_{\text {opt }}(a, b)$, from the

341 number $N=13$ of data-points (size of Table 1 rather than the number of fish), and from the

342 number $K$ of optimized parameters:

343

(6) $A I C_{c}($ model $)=N \cdot \ln \left(\frac{S S E(\text { model })}{N}\right)+2 \cdot K+\frac{2 \cdot K \cdot(K+1)}{N-K-1}$

344

(7) $\operatorname{prob}($ model $)=\frac{e^{-\Delta / 2}}{1+e^{-\Delta / 2}}$, where $\Delta=A I C($ model $)-A I C($ best fitting model $)>0$.

The Akaike weight prob compares a model with the best fitting model in terms of the least $A I C_{c}$ : Its Akaike weight prob(model) is the probability that this model is true (assuming that one of the two models is true); the maximal Akaike weight is 50\%. (This interpretation is based on the assumption of normally distributed errors. As the data were average values of large samples, this assumption was justified. However, the Akaike weight may also be interpreted as just another measure of the goodness of fit to the data; see below. Such an interpretation does not need the assumption of a normal distribution.)

Technically, the application of the above criteria requires that two distinctions are made: First, the differential equations (1) and (2) that set the general framework for this study need to be distinguished from the different growth models that may or may not assume specific values for the exponent-pair. Thereby, each grid point defined a concrete model of type (1) with an assumed exponent-pair $(a, b)$; e.g. logistic model with $(a, b)=(1,2)$. The (other) model parameters $\left(m_{0}, p, q\right)$ were optimized (data fitting). However, the third round of optimization in addition sought for optimal exponents, referring to the general Bertalanffy-Pütter model and the general Gompertz model, respectively. Thereby, the $A I C$ of models with assumed exponent-pairs was computed with $K=4$ (as implicitly also $S S E$ was optimized). The $A I C$ of the general 
362 respectively, as also the exponents were optimized. Owing to this penalty for additional

363 parameters, the best fitting model in terms of the least SSE could have a higher (worse) $A I C$ than

364 other models. Second, we interpreted the Akaike weights in two ways. If the $A I C$ was computed

365 with the above explained correct number of parameters, the Akaike weights might be interpreted

366 in the usual way as probabilities about the truth of a model. However, we also used the Akaike

367 weights with an incorrect number of parameters, assuming $K=4$ for all models; i.e. also the

368 models with optimized exponents were treated as if these exponents were given in advance. For

369 this application, the Akaike weight was merely a measure of the good fit (low $S S E$ ) that was

370 comparable across different data-sets, but not a probability of truth.

371 We use the second interpretation to define acceptability and near-optimality (Renner-Martin et

372 al., 2018): A model defined from an assumed exponent-pair $(a, b)$ has an acceptable fit, if in

373 comparison to the optimal exponent-pair its Akaike-weight is 2.5\% or higher (i.e. the lowest 5\%

374 of Akaike-weights are deemed as inacceptable), whereby all Akaike-weights are computed with

$375 K=4$ (assuming that the optimal exponent-pair was given in advance). The exponent-pairs with

376 an acceptable fit define the region of near-optimality. Using some algebra, this definition is

377 equivalent to the following condition in terms of $S S E, N$ and $t$ (the above threshold), which

378 defines acceptability by a maximal percentage (dependent on $N$ ), by which the optimal $S S E$ may

379 be exceeded (e.g. for $N=13$ and $t=0.025=2.5 \%$, an excess of $75.7 \%$ is acceptable):

380
(8) $\frac{\operatorname{SSE}(\text { model })}{\operatorname{SSE}(\text { best fitting model })}<1.757=\left(\frac{1}{t}-1\right)^{2 / N}$.

381 Figure 4 (all Akaike-weights computed with $K=4$ ) shows that amongst generalized von

382 Bertalanffy models (defined by exponent pairs with $b=1$ ), the comparison with the best-fitting

383 model did affect the Akaike weights only slightly. For instance, for the Bertalanffy pair the 
384 Akaike weight was reduced from 36\% (comparison with the optimal exponent $a$, assuming $b=1$ )

385

386

387

388

389

390

391

392

393

394

395

396

397 398

399

400

401

402

403

404

405

406

to $34 \%$ (comparison with the best-fitting exponent-pair). For lower Akaike-weights the reduction

was even smaller, whence the Akaike-weights could not be pushed below the $2.5 \%$ threshold.

Thus, despite the comparison with the overall optimal Bertalanffy-Pütter model, for the class of generalized von Bertalanffy models $(b=1)$ all exponents $0 \leq a<1$ were acceptable.

Figure 5 illustrates, how this variability extended into two dimensions (the dimensions referring to the number of considered exponents). The green area represents exponent-pairs, whose $A I C$ was below the correct $A I C$ of the best-fitting model. (Thereby, $A I C$ for given exponent-pairs was computed with $K=4$, while the $A I C$ for best fitting Gompertz-type model was computed with $K$ $=5$, whence there was a penalty.) The red area represents additional exponent-pairs, whose fit was deemed as acceptable in the meaning above (Akaike weight of $2.5 \%$ or higher, using $K=4$ also for the best fitting model). The red area was bounded, meaning that in two dimensions not all exponent-pairs were acceptable.

The following examples illustrate these concepts. In Figure 2, the best fit in terms of SSE was achieved by the optimal exponent-pair, followed by the von Bertalanffy-pair, while logistic growth had the poorest fit. However, owing to the penalty in the definition of AIC for using more parameters, the von Bertalanffy exponent-pair was in the green region of Figure 5. Therefore, when choosing between the von Bertalanffy and the best fitting exponent-pair, the criterion of parsimony would recommend to select the former one. The logistic exponent-pair was outside the red or green regions of Figure 5, whence this fit was deemed as not acceptable, although in Figure 2 it still appeared to be reasonable. Summarizing, when comparing these exponent pairs, the von Bertalanffy-pair would be selected as parsimonious; the logistic pair would be refuted due to its poor fit; and the optimal pair would be refuted, as its $10 \%$ reduction of $S S E_{\text {opt }}$ (in 
407 comparison to the von Bertalanffy exponent-pair) did not justify the optimization of an

408 additional parameter. Figure 6 indicates that equations (1) and (2) may indeed result in overfit

409 due to the optimization of too many parameters. Using model (2) together with the optimal

410 exponent, it plots the region of the 'other parameters' $\left(m_{0}, p, q\right)$, where $S S E$ was bounded by $10^{7}$

411 (ca. 500 times the least SSE). Despite this large SSE, the region was extremely thin, suggesting

412 some relation between the parameters. This indicates that a subclass of the Bertalanffy-Pütter

413 model using fewer parameters may provide the same fit and therefore suffice for the modeling of

414 growth. There remains the problem to find such a subclass that in addition is empirically 415 meaningful.

\section{CONCLUSION}

417 The paper conducted a case study about Bertalanffy-Pütter exponent-pairs $(a, b)$ for fish. It was

418 based on mass-at-age data of Walleye (Sander vitreus). Comparing the von Bertalanffy

419 exponent-pair model with the general Bertalanffy-Pütter and Gompertz models, the general 420 models reduced bias in growth estimates $(S S E)$ but increased model complexity. However, there 421 was a large region of near-optimal exponent-pairs, amongst them the von Bertalanffy exponent422 pair. Therefore, parsimonious model selection $(A I C)$ confirmed the established practice to 423 describe growth in term of the Bertalanffy models (VBGF for length, the von Bertalanffy 424 exponent-pair for mass). However, there are purposes, where the predictive power of models and the good fit of the model curve to the data is more important, than simplicity. For example, if a 426 model is used to justify policy recommendations on the basis of certain data, such as size-based 427 catch-limitations in fishery stock assessments, it is of crucial importance that the model curve fits 428 well to the data. Further, even in cases where the Bertalanffy-exponent pair is the most 429 parsimonious model, it may not be true, as biological arguments (e.g. about metabolism) may 
430 support different exponent-pairs (e.g. the models mentioned in the introduction). Thus, Pauly

431 (1981) recommended to use the class of generalized von Bertalanffy models $(b=1$ and the

432 exponent $a$ is free). We go one step further and recommend the use of the Bertalanffy-Pütter

433 model (and of its limit case, the generalized Gompertz model) in natural resources management

434 and other contexts that rely on careful quantitative assessments.

435 With respect to future research, we speculate that for the best fitting parameters there may exist additional relations, whence optimization might be further constrained by some functional relationship between the parameters. This would define a subclass of Bertalanffy-Pütter models

for the evaluation of the goodness of fit.

\section{ACKNOWLEDGEMENTS}

The authors appreciate the insightful comments by two reviewers that improved this paper substantially.

\section{REFERENCES}

Akaike, H. (1974). A New Look at the Statistical Model Identification. IEEE Trans. Automatic Control, 19, 716723.

Apostolidis, C.; Stergiou, K.I. (2014). Estimation of growth parameters from published data for several Mediterranean fishes. Journal of Applied Ichthyology, 30, 189-194.

Bertalanffy, L.v. (1957). Quantitative laws in metabolism and growth. Quarterly Reviews of Biology, 32, 217-231.

Burden, R.L.; Faires, J.D. (1993). Numerical Analysis, PWS Publishing Co. Boston, MA.

Burnham, K.P.; Anderson, D.R. (2002). Model Selection and Multi-Model Inference: A Practical InformationTheoretic Approach. Springer, Berlin.

Cailliet, G.M.; Smith, W.D.; Mollet, H.F.; Goldman, K.J. (2006). Age and growth studies of chondrichthyan fishes: The need for consistency in terminology, verification, validation, and growth function fitting. Environmental Biology of Fish, 77, 211-228.

Carvalho, E.P.d.; Santoro, K.R. (2007). On Starting Values for Parameters of Nonlinear Growth Functions with Application in Animal Science. Journal of Concrete and Applicable Mathematics, 5, 133-142. 
458

459

460

461

462

463

464

465

466

467

468

469

470

471

472

473

474

475

476

477

478

479

480

481

482

483

484

485

486

487

488

489

490

491

492

493

494

495

496

497

498

499

500

501

Cedersund, G.; Samuelsson, O.; Ball, G.; Tegner, J.; Gomez-Cabrero, D. (2015). Optimization in Biology. Parameter Estimation and the Associated Optimization Problem. In Gomez-Cabrero, D.; Geris, L. (editors). Uncertainty in Biology, Springer Verlag, Heidelberg, Germany.

Dziak, J.J.; Coffman, D.L.; Lanza, S.T., Li, R. (2017). Sensitivity and specificity of information criteria. PeerJ Preprints, 5, published online DOI: 10.7287/peerj.preprints.1103v3.

Enberg, K.; Dunlop, E.S.; Jørgensen, C. (2008). Fish growth. In: Jørgensen, S.E.; Fath, B.D. (eds.) Encyclopedia of ecology. Elsevier, Oxford, 2008, pp. 1564-1572.

Forsgen, A.; Gill, P.E.; Wright, M.H. (2002). Interior point methods for nonlinear optimization. SIAM Review, 44, $525-597$

Froese, R.; Pauly, D. (2018). FishBase data base: www.fishbase.org, last accessed in June 2018.

Gompertz, B. (1832). On the Nature of the Function Expressive of the Law of Human Mortality, and on a New Mode of Determining the Value of Life Contingencies. Phil. Transactions Royal Society London, 123, 513-585.

Karmakar, N. (1984). A new polynomial-time algorithm for linear programming. Combinatorica, 4, 373-395.

Killen, S.S., Atkinson, D., Glazier, D.S. (2010). The intraspecific scaling of metabolic rate with body mass in fishes depends on lifestyle and temperature. Ecology Letters, 13, 184-193.

Leader, J. J. (2004). Numerical Analysis and Scientific Computation. Addison-Wesley, Boston.

Manabe, A.; Yamakawa, T.; Ohnishi, S.; Akamine, T.; Narimatsu, Y.; Tanaka, H.; Funamoto, T.; Ueda, Y.; Yamamoto, T. (2018). Anovel growth function incorporating the effects of reproductive energy allocation. PLoS ONE, 13, published online, DOI: 10.1371/journal.pone.0199346.

Marusic, M.; Bajzer, Z. (1993). Generalized two-parameter equations of growth. Journal of Mathematical Analysis and Applications, 179, 446-462.

Mildenberger, T.K.; Taylor, M.H.; Wolff, M. (2017). TropFishR: a R-package for fisheries analysis with lengthfrequency data. Methods in Ecology and Evolution, doi: 10.1111/2041-210X.12791.

Motulsky, H.; Christopoulos, A. (2003). Fitting Models to Biological Data Using Linear and Nonlinear Regression: A Practical Guide to Curve Fitting. Oxford Univ. Press, Oxford.

Ogle, D. (2018). R for Fisheries Analysis, derekogle.com, last accessed in June 2018.

Ogle, D.; Iserman, D.A. (2017). Estimating Age at a Specified Length from the von Bertalanffy Growth Function. North American Journal of Fisheries Management, 37, 1176-1180.

Ohnishi, S.; Yamakawa, T; Akamine, T. (2014). On the analytical solution for the Pütter-Bertalanffy growth equation. J. Theoretical Biology, 343, 174-177.

Pauly, D. (1981). The relationship between gill surface area and growth performance in fish: a generalization of von Bertalanffy's theory of growth. Reports on Marine Research (Berichte der deutschen wissenschaftlichen

Kommission für Meeresforschung), 28, 25-282.

Pütter, A. (1920). Studien über physiologische Ähnlichkeit. VI. Wachstumsähnlichkeiten. Pflüg. Archiv der Gesamten Physiologie, 180, 298-340.

Renner-Martin, K.; Brunner, N.; Kühleitner, M.; Nowak, W.G.; Scheicher, K. (2018). On the exponent in the Von Bertalanffy growth model. PeerJ, 6, published online, DOI: 10.7717/peerj.4205.

Richards, F.J. (1959). A Flexible Growth Function for Empirical Use, Journal of Experimental Botany, 10, 290-300.

Shi, P.-J.; Ishikawa, T.; Sandhu, H.S.; Hui, C.; Chakraborty, A.; Jin, X.-S.; Tachihara, K.; Li, B.-L. (2014). On the 3/4-exponent van Bertalanffy equation for ontogenetic growth. Ecological Modelling, 276, 23-28.

Seaborn, J.B. (2013). Hypergeometric functions and their applications, VIII. Springer Science \& Business Media, New York.

Strathe, A.B.; Danfær, A.; Sørensen, H.; Kebreab, E. (2010). A multilevel nonlinear mixed-effects approach to model growth in pigs. Journal of Animal Science, 88, 638-649. 
502 Ursin, E. (1979). Principles of growth in fishes. Symp. Zool. Soc. London, 44, 63-87.

503 Verhulst, P.F. (1838). Notice sur la loi que la population suit dans son accroissement, Correspondence

504 Mathematique et Physique (Ghent), 10, 113-121.

505 Vidal, R.V.V. (1993). Applied simulated annealing. Lecture notes in economics and mathematical systems.

506 Springer-Verlag, Berlin.

507 West, G.B.; Brown, J.H.; Enquist, B.J. (2001). A general model for ontogenetic growth. Nature, 413, 628-631.

508 Wilson, K.L.; Matthias, B.G.; Barbour, A.B.; Ahrens, R.N.M.; Tuten, T.; Allen, M.S. (2015). Combining samples

509 from multiple gears helps to avoid fishy growth curves. North American Journal of Fisheries Management, 35,

$510 \quad 1121-1131$.

511 White, C.R. (2010). Physiology: There is no single p. Nature, 464, 691-693.

512

513

514 


\section{Table and Figure Captions}

516 Table 1. Average weight-at-age (rounded) for male Walleye, based on ca. 20,000 age-weight data points (rounded to 517 one decimal for the ease of presentation; the computations of the paper used data rounded to three decimals).

518 Table 2. Optimal parameters for selected models.

519 Figure 1. Weight-at-age and average weight (red dots) of male Walleye from Lake Erie.

520 Figure 2. Comparison with the data of the growth curve using the Bertalanffy exponent-pair (red), the logistic 521 exponent pair (blue) and of the best fitting growth curve (black); parameter values as in Table 2.

522 Figure 3. Contour plot of the optimal SSE on a grid of exponent-pairs with distance 0.01 between adjacent points 523 and for each exponent $a$, plot of the exponent-pair with smallest SSE (black dots).

524 Figure 4. Plot of the Akaike weights for exponent-pairs with $b=1$, using the least $A I C$ amongst generalized

525 Bertalanffy-models (red) and the least $A I C$ amongst all considered models (blue); all $A I C$ s using $K=4$.

526 Figure 5. Plot of the grid points $a<b$ with AIC below AIC of the best fitting model (green; the AIC of the best fitting model was higher due to the penalty for an additional parameter) and with acceptable fit (red). The Bertalanffy and the logistic exponent-pairs are displayed in yellow.

529 Figure 6. Plot of part of the region of exponents $m_{0}, p, q$ for model (2) with the optimal exponent $a=0.686028$, where SSE does not exceed $10^{7}$. 


\section{Table $\mathbf{1}$ (on next page)}

Average weight-at-age (rounded) for male Walleye, based on ca. 20,000 age-weight data points (rounded to one decimal for the ease of presentation; the computations of the paper used data rounded to three decimals) 
1 Table 1

2 Average weight-at-age (rounded) for male Walleye, based on ca. 20,000 age-weight data points (rounded to one

3 decimal for the ease of presentation; the computations of the paper used data rounded to three decimals)

4

\begin{tabular}{crrl}
\hline Age (years) & Weight (gram) & Class size & Comment \\
\hline 0 & 192.1 & 14 & \\
1 & 423.7 & 4009 & \\
2 & 761.8 & 5181 & \\
3 & 1018.0 & 3870 & \\
4 & 1221.6 & 2262 & \\
5 & 1442.8 & 1519 & \\
6 & 1644.5 & 1471 & \\
7 & 1802.0 & 690 & \\
8 & 1880.7 & 446 & \\
9.5 & 1895.3 & 430 & classes $9+10$ \\
11 & 1982.6 & 105 & \\
12.4 & 2140.4 & 104 & classes $12+13$ \\
15.3 & 2228.5 & 65 & classes 14-20 \\
\hline
\end{tabular}

5 
Table 2 (on next page)

Optimal parameters for selected models

${ }^{*} 1^{\text {st }}$ and $3^{\text {rd }}$ refer to the initial and final rounds of optimization 
1 Table 2

2 Optimal parameters for selected models

3

\begin{tabular}{cccccccc} 
Model & Comment $^{*}$ & $\mathrm{a}$ & $\mathrm{b}$ & $\mathrm{m}_{0}$ & $\mathrm{p}$ & $\mathrm{q}$ & SSE \\
\hline Bertalanffy & $1^{\text {st }}(\mathrm{a}, \mathrm{b}$ given $)$ & $2 / 3$ & 1 & 203.8 & 11.2 & 0.86 & 23,709 \\
logistic & $1^{\text {st }}(\mathrm{a}, \mathrm{b}$ given $)$ & 1 & 2 & 301.716 & 0.528051 & 0.000253611 & 72,283 \\
optimal & $3^{\text {rd }}($ a optimized $)$ & 0.686028 & $=\mathrm{a}$ & 175.67 & 21.3148 & 2.76054 & 21,286 \\
\hline
\end{tabular}

$4{ }^{*} 1^{\text {st }}$ and $3^{\text {rd }}$ refer to the initial and final rounds of optimization

5 
Figure 1

Weight-at-age and average weight (red dots) of male Walleye from Lake Erie

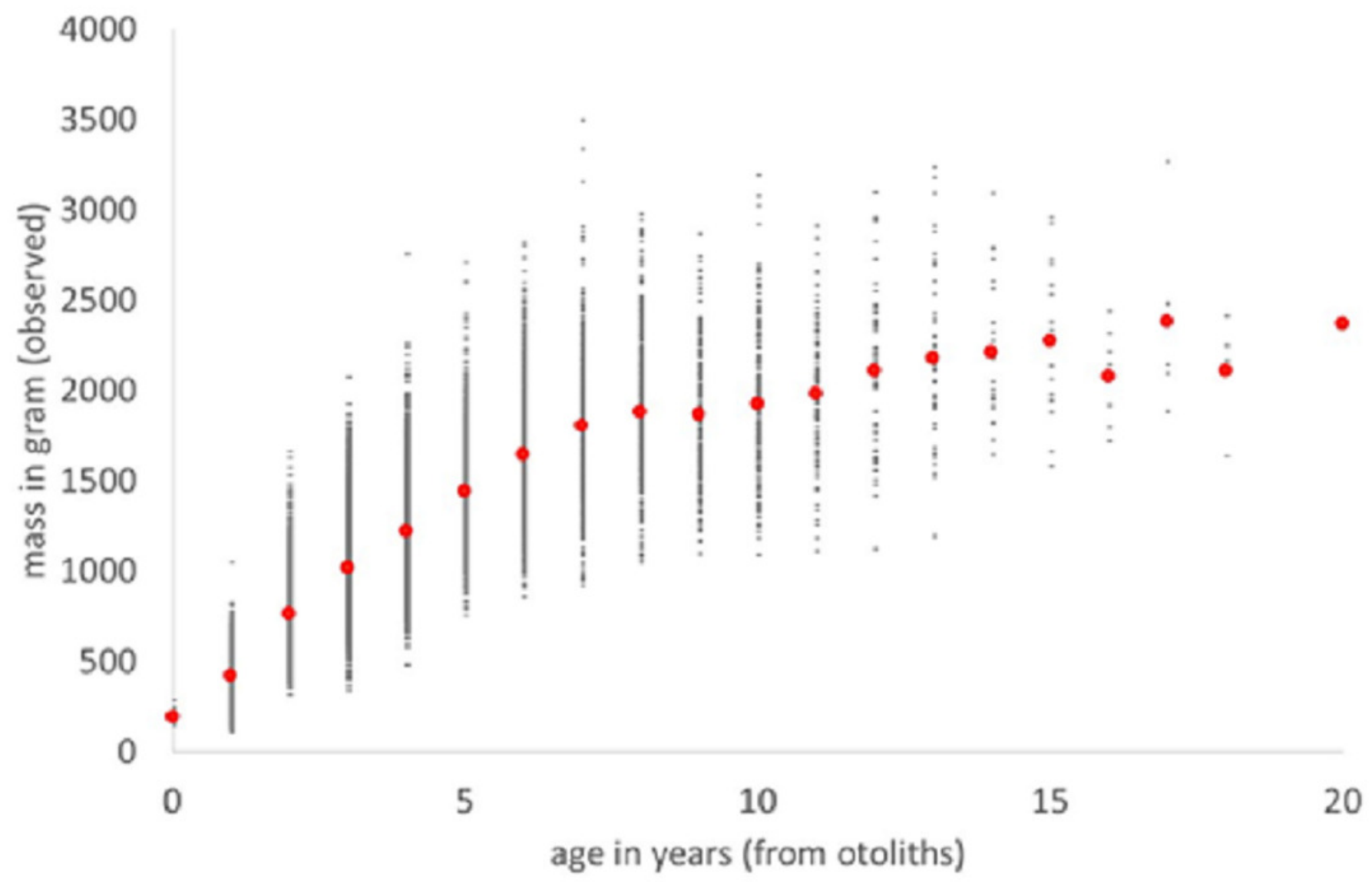


Figure 2

Comparison with the data of the growth curve using the Bertalanffy exponent-pair (red), the logistic exponent pair (blue) and of the best fitting growth curve (black); parameter values as in Table2.

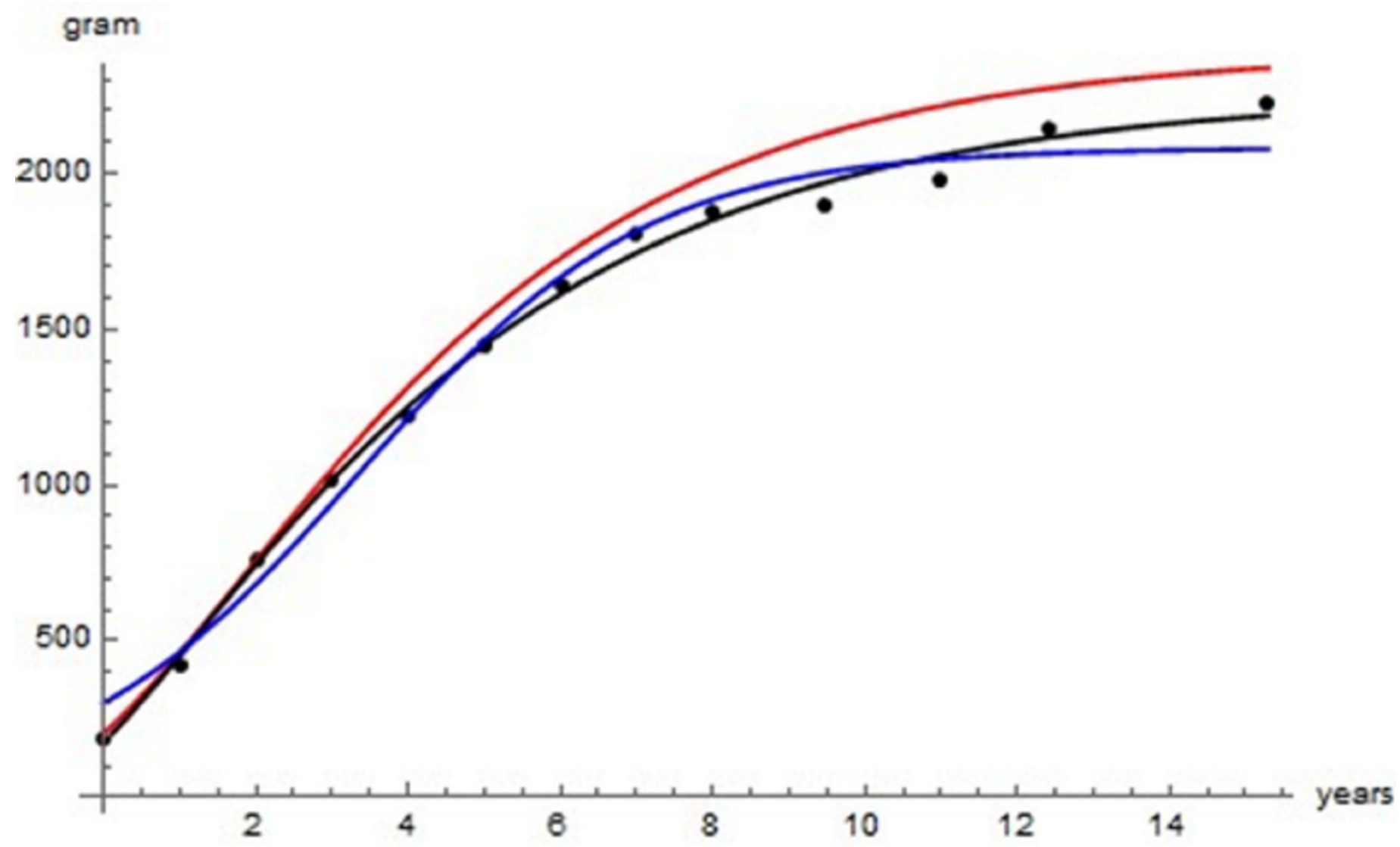




\section{Figure 3}

Contour plot of the optimal SSE on a grid of exponent-pairs with distance 0.01 between adjacent points and for each exponent a, plot of the exponent-pair with smallest SSE (black dots).

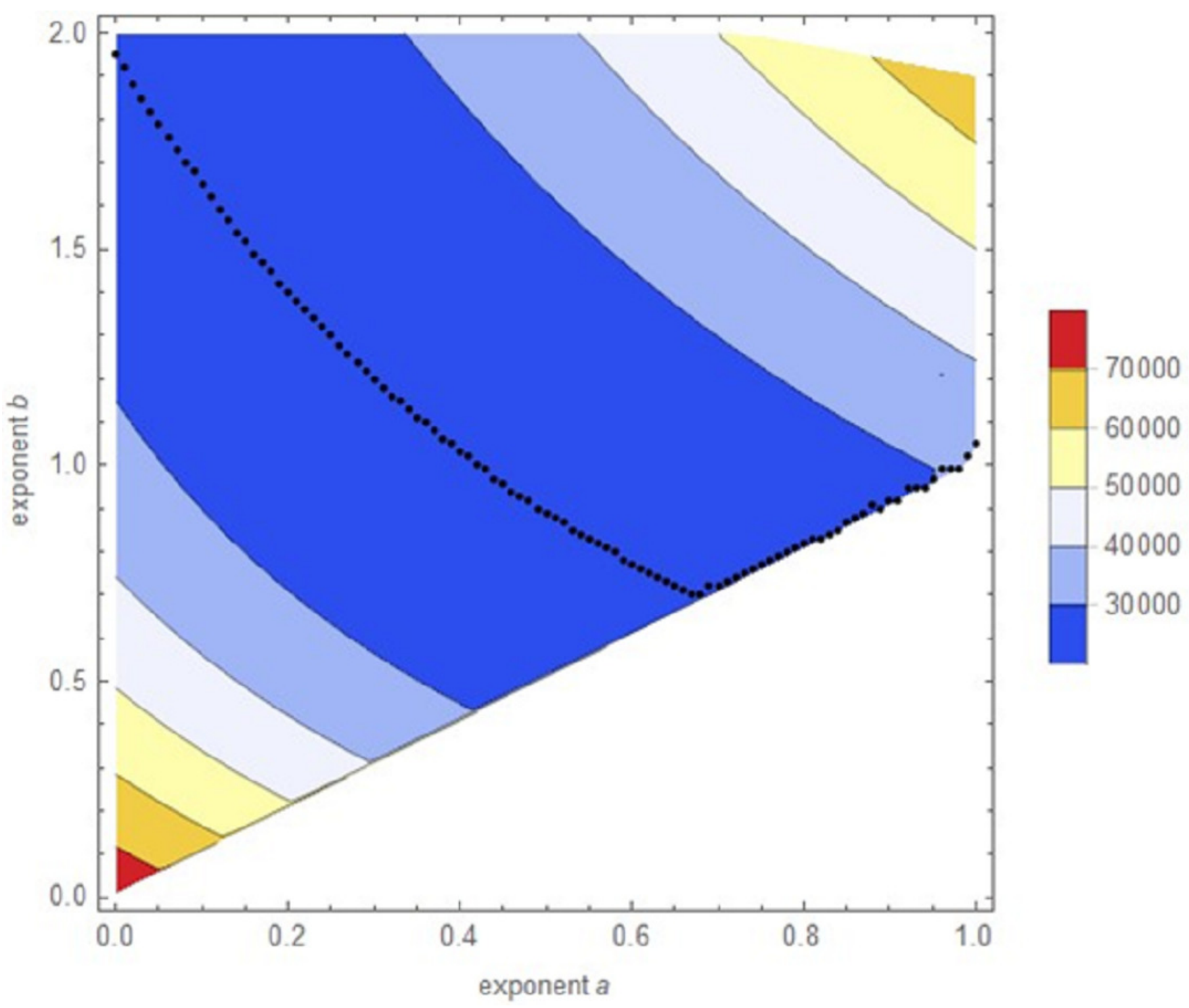


Figure 4

Plot of the Akaike weights for exponent-pairs with $b=1$, using the least AIC amongst generalized Bertalanffy-models (red) and the least AIC amongst all considered models (blue); all AICs using $\mathrm{K}=4$

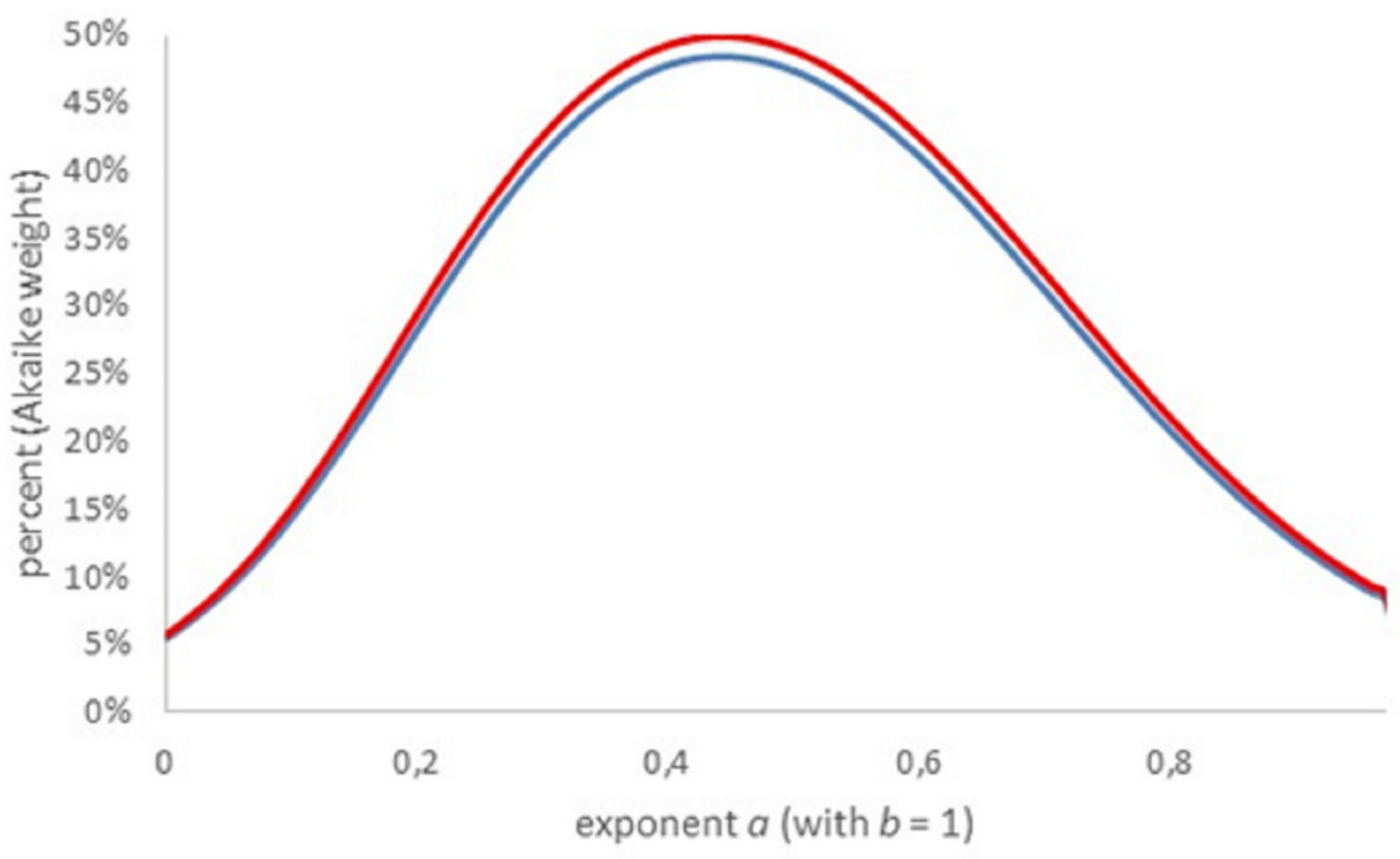




\section{Figure 5}

Plot of the grid points $a<b$ with AIC below AIC of the best fitting model (green; the AIC of the best fitting model was higher due to the penalty for an additional parameter) and with acceptable fit (red). The Bertalanffy and the logistic exponent-pairs

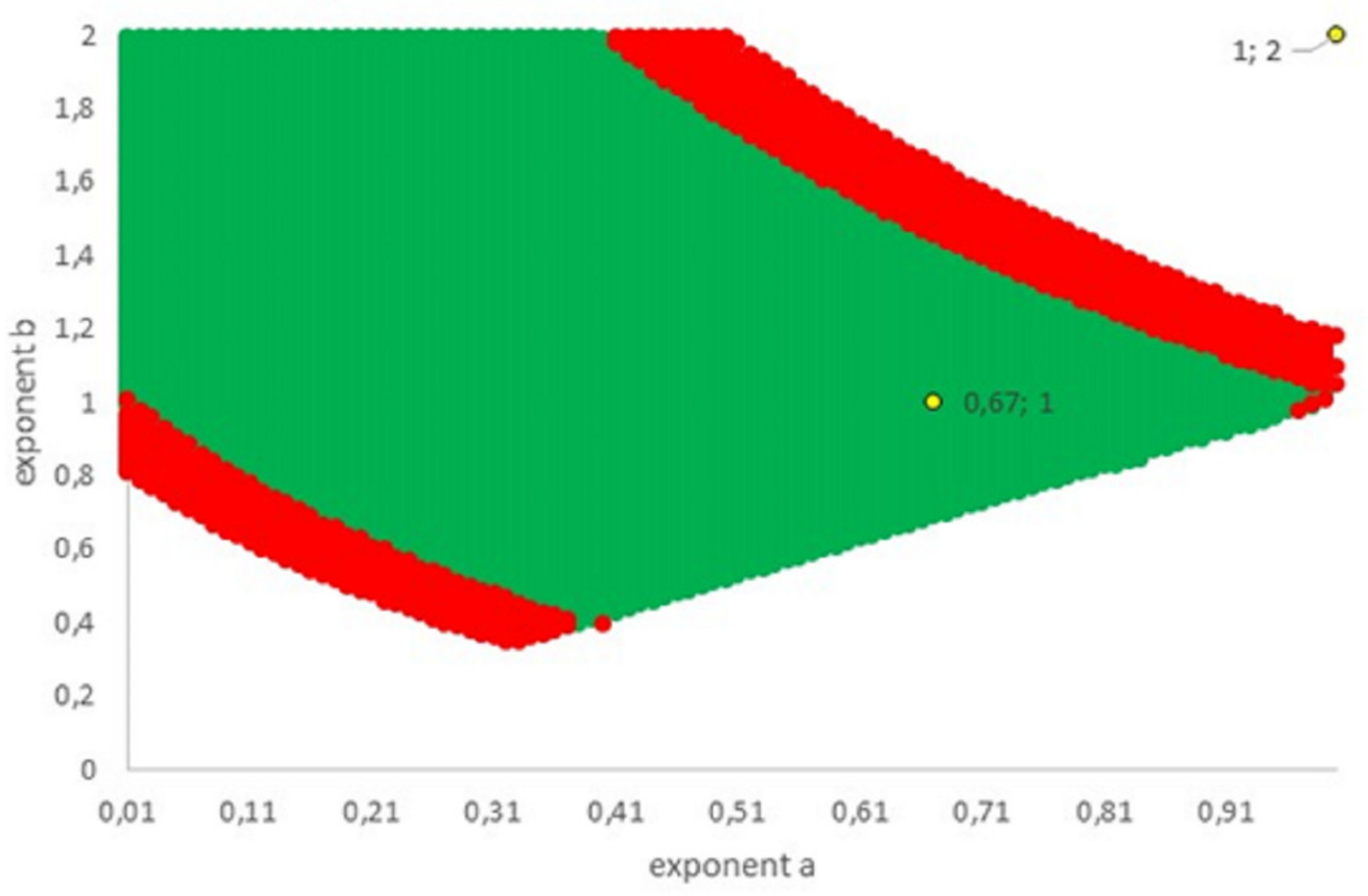


Figure 6

Plot of part of the region of exponents $m_{-} 0 p$, q for model (2) with the optimal exponent $\mathrm{a}=0.686028$, where SSE does not exceed 10^7. 


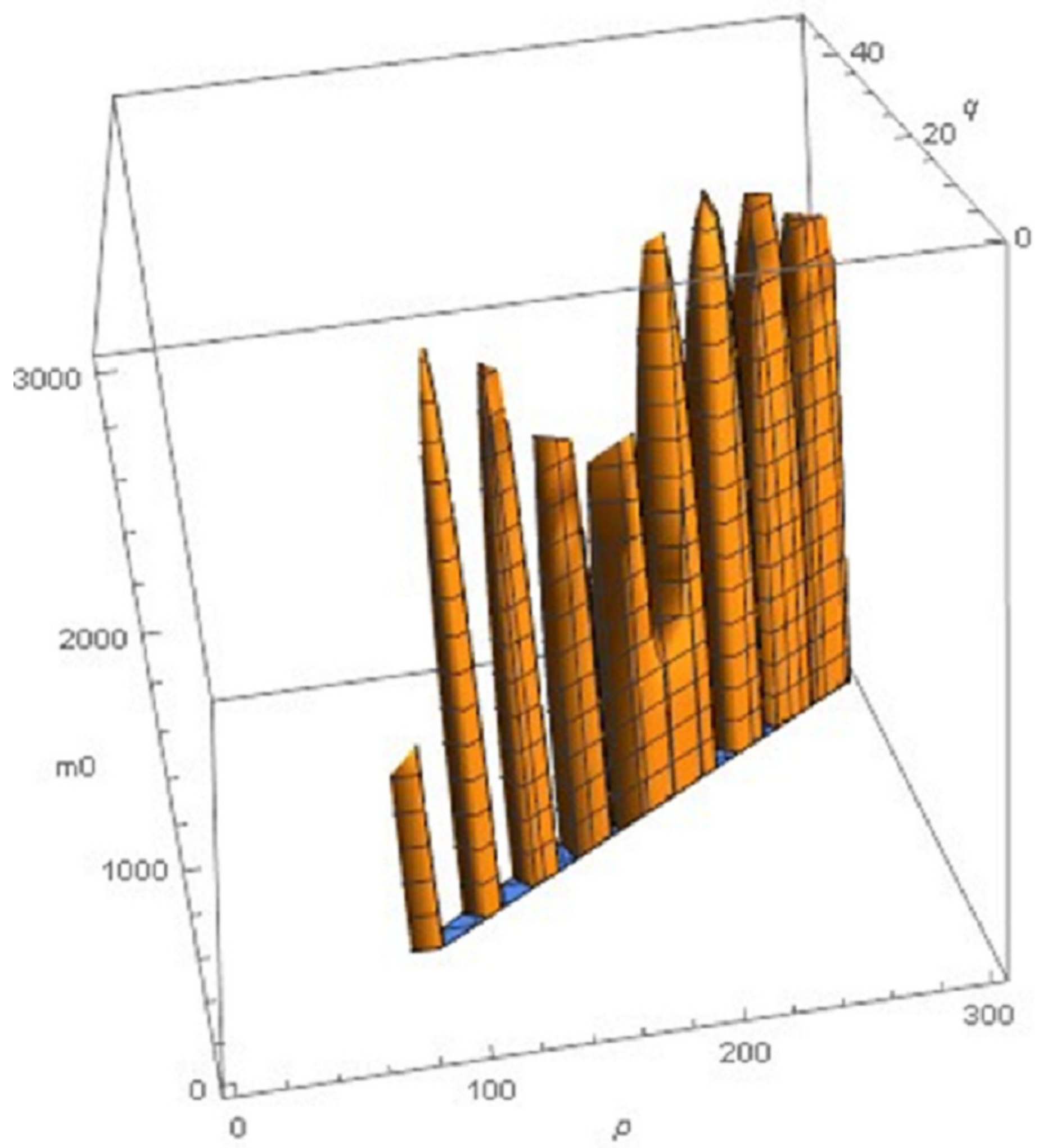

